\title{
Immune checkpoint inhibitor in liver cancer-unique regional differences
}

\author{
Shuntaro Obi, Takahisa Sato, Shinpei Sato \\ Department of Internal Medicine, Teikyo University Chiba Medical Center, Chiba, Japan \\ Correspondence to: Shuntaro Obi, MD, PhD. Department of Internal Medicine, Teikyo University Chiba Medical Center, 3426-3 Anesaki, Ichihara, \\ Chiba 299-0111, Japan. Email: obis@med.teikyo-u.ac.jp. \\ Comment on: Yau T, Hsu C, Kim TY, et al. Nivolumab in advanced hepatocellular carcinoma: Sorafenib-experienced Asian cohort analysis. J Hepatol \\ 2019;71:543-52.
}

Submitted Apr 17, 2020. Accepted for publication May 07, 2020.

doi: $10.21037 / \mathrm{atm}-20-3378$

View this article at: http://dx.doi.org/10.21037/atm-20-3378

HCC, which accounts for most of the liver cancer, a globally common disease that ranks fourth in cancer-related deaths (1). Risk factors for HCC include viral hepatitis, alcoholic hepatitis, nonalcoholic steatohepatitis, and exposure to aflatoxin (2). These risk factors vary from region to region due to uneven distribution of hepatitis virus, religion, economic problems, environment and hygiene (3). These HCC risk factors are theoretically preventable but practically difficult. Therefore, the number of HCC patients depends on the amount of risk factors in each region. Regional genetic differences in HCC also affect therapeutic efficacy. It is unclear whether this genetic difference is due to etiology, environment, or race. Surveillance by setting high-risk group is effective for early detection of HCC. However, due to social issues including the economy, medical resources also have a serious regional disparity. In areas with inadequate medical resources, HCC cases are on the rise and are detected in advanced stages. The treatment guidelines for HCC dictate the recommended treatment by a staging system consisting of liver function factors and tumor factors. HCC treatment guidelines have also been taken regional characteristics into consideration (4-7). Treatment methods also have regional characteristics. Even the standard treatment, including surgical resections, local ablation, and TACE, recommended in the guidelines vary in frequency and timing of selection by region (3). In addition, the diversity of treatment modalities by region is even greater. Large regional differences in HCC itself, background, and management are thought to have a major impact on the therapeutic effects and adverse events of systemic therapy. This article discusses regional differences in the therapeutic efficacy of systemic therapy.

The almost 10 years, sorafenib, an anti-angiogenic multi-kinase inhibitor, was the only drug with strong evidence to demonstrate therapeutic efficacy against for advanced HCC (8). Two years ago, a new agents lenvatinib, an inhibitor of VEGF receptors 1-3, FGF receptors 1-4, PDGF receptor $\alpha$, RET, and KIT, as first-line setting have demonstrated activity in phase III studies (9). In secondline setting, for more than a decade, many clinical trials were done worldwide, but with disappointing negative results. However, in recent years, the therapeutic efficacy of several new drugs has been demonstrated. These are anti-angiogenic multi-kinase inhibitors regorafenib, cabozantinib, and the monoclonal antibody ramucirumab (10-12). Interestingly, there were regional differences in these drug treatment outcomes. A lenvatinib phase III clinical study compared overall survival (OS) in the AsiaPacific and Western regions. OS, which is the primary endpoint in this trial, was found to be effective in the Asian region. As a result, the hazard ratio (HR) for OS for the Asia-Pacific region was 0.86, while the HR for the Western was 1.08. Progression free survival as secondary end point was effective in both Asia-pacific region with HR of 0.61 and western with 0.81 , but was more prominent in Asiapacific region (2). Although the cause of region difference is unknown, lenvatinib may have been taken for an enough period because the adverse events were controlled due to dose-controlled by body weight. Region differences in treatment efficacy were also observed in ramucirumab, 
human IgG1 monoclonal antibody which inhibits ligand activation of VEGFR2. A phase 3 trial of ramucirumab (REACH-2 trial) was conducted in OS as a primary endpoint in second-line patients after Sorafenib treatment with AFP of $400 \mathrm{ng} / \mathrm{mL}$ or more. In this study, OS was compared between region 1 (America, Europe, Australia, and Israel), region 2 (Asia excluding Japan), and region 3 (Japan). The hazard ratio of OS was 0.75 for the region 1 , 0.83 for the region 2, and 0.65 for the region 3, respectively. Regional differences were also more notably observed for PFS with hazard risks of $0.49,0.55$ and 0.28 in region $1 v s$. region $2 v s$. region 3, respectively (12). Interestingly enough, the HR for OS and PFS are lower in the order of region 3 (Japan), region 1 (almost Western), and region 3 (Asia excluding Japan). Although Japan geographically belongs to Asia, it is quite different in terms of therapeutic effect on hepatocellular carcinoma by ramucirumab. In contrast, a cabozantinib phase III clinical trial was more effective in the non-Asia region, with an OS HR of 1.01 in the Asia region versus a hazard ratio of 0.71 in the non-Asia region (11). These facts prove that the difference in therapeutic effects by region is not limited to racial differences. It is necessary to discuss the cause of further regional differences in the future.

Recently, phase II or III clinical trials of the antiprogrammed death-1 (PD-1) monoclonal antibodies drugs (nivolumab and pembrolizumab) reported safety and efficacy in patients with advanced hepatocellular carcinoma $(13,14)$. PD-1 monoclonal antibodies, also have regional difference in treatment efficacy. In a phase III clinical trial of pembrolizumab, OS was compared in Asia (excluding Japan) and non-Asia regions (including Japan). HR of OS in the Asia region was 0.59 compared to 0.86 in the non-Asia. PFS also showed regional difference (15). In this study, OS and PFS did not reach statistical significance below certain criteria, but there were regional differences in treatment efficacy. In PFS, the HCV group had the lowest HR (HBV: $0.70, \mathrm{HCV}: 0.46$, and uninfected: 0.75 ). This fact suggests that factors other than viruses are involved in regional differences of OS in pembrolizumab.

On the other hand, for nivolumab, phase $1 / 2$, noncomparative, dose escalation and expansion trial (CheckMate 040) was performed (13). In this study, regional difference of treatment efficacy was not investigated. As mentioned above, there are large regional differences in the therapeutic effect of HCC. It was very important to analyze regional differences in the therapeutic effect of nivolumab. Therefore, Yau et al. evaluated the safety and efficacy of nivolumab in the Asian cohort (16). Unfortunately, the relatively small sample size made it impossible to directly compare the Asian and non-Asian cohorts. Because of the comparison between the Asian cohort and the ITT population, the result has some bias in this study. The Nivolumab phase II trial showed median duration of response 9.7 months in the Asian cohort vs. 19.4 in ITT population. Regional difference also showed response PD $47 \%$ in the Asian cohort vs. $39 \%$ in ITT population. However, median overall survival was similar between the Asian cohort (14.9 months) vs. the ITT population (15.1 months). The safety profile was also similar both populations (16). Asian cohort had almost the same median overall survival, although the median duration of response was less than half that of the ITT population. It is necessary to discuss why such results are obtained and what is affecting the OS.

Patients of HCC are a very heterogeneous population. The heterogeneity may be due to regional differences. These differences are such as etiology (hepatitis virus, alcohol, steatosis and genetic mutations), clinical presentation (patients in the Asian region tend to be more advanced) and management (Asian patients tend to receive more prior therapies). The fusion of these differences can lead to variations in the clinical efficacy and safety of therapeutic agents.

In conclusion, a thorough understanding of the regional differences in therapeutic efficacy in drug treatment is important in determining the most effective treatment strategy.

\section{Acknowledgments}

Funding: None.

\section{Footnote}

Provenance and Peer Review: This article was commissioned by the editorial office, Annals of Translational Medicine. The article did not undergo external peer review.

Conflicts of Interest: All authors have completed the ICMJE uniform disclosure form (available at http://dx.doi. org/10.21037/atm-20-3378). All authors have no conflicts of interests to declare.

Ethical Statement: The authors are accountable for all aspects of the work in ensuring that questions related 
to the accuracy or integrity of any part of the work are appropriately investigated and resolved.

Open Access Statement: This is an Open Access article distributed in accordance with the Creative Commons Attribution-NonCommercial-NoDerivs 4.0 International License (CC BY-NC-ND 4.0), which permits the noncommercial replication and distribution of the article with the strict proviso that no changes or edits are made and the original work is properly cited (including links to both the formal publication through the relevant DOI and the license). See: https://creativecommons.org/licenses/by-nc-nd/4.0/.

\section{References}

1. Torre LA, Bray F, Siegel RL, et al. Global cancer statistics, 2012. CA Cancer J Clin 2015;65:87-108.

2. Villanueva A. Hepatocellular Carcinoma. N Engl J Med 2019;380:1450-62.

3. Choo SP, Tan WL, Goh BKP, et al. Comparison of hepatocellular carcinoma in Eastern versus Western populations. Cancer 2016;122:3430-46.

4. Bruix J, Sherman M. American Association for the Study of Liver Diseases: management of hepatocellular carcinoma: an update. Hepatology 2011;53:1020-2.

5. European Association For The Study Of The Liver; European Organisation For Research And Treatment Of Cancer. EASL-EORTC Clinical Practice Guidelines: management of hepatocellular carcinoma. J Hepatol 2012;56:908-43.

6. Omata M, Lesmana LA, Tateishi R, et al. Asian Pacific Association for the study of the liver consensus recommendations on hepatocellular carcinoma. Hepatol Int 2010;4:439-74.

7. Kudo M, Matsui O, Izumi N, et al. JSH consensusbased clinical practice guidelines for the management of hepatocellular carcinoma: 2014 update by the Liver Cancer Study Group of Japan. Liver Cancer 2014;3:458-68.

Cite this article as: Obi S, Sato T, Sato S. Immune checkpoint inhibitor in liver cancer-unique regional differences. Ann Transl Med 2020;8(21):1336. doi: 10.21037/atm-20-3378
8. Llovet JM, Ricci S, Mazzaferro V, et al. Sorafenib in advanced hepatocellular carcinoma. N Engl J Med 2008;359:378-90.

9. Kudo M, Finn RS, Qin S, et al. Lenvatinib versus sorafenib in first-line treatment of patients with unresectable hepatocellular carcinoma: A randomised phase 3 noninferiority trial. Lancet 2018;391:1163-73.

10. Bruix J, Qin S, Merle P, et al Regorafenib for patients with hepatocellular carcinoma who progressed on sorafenib treatment (RESORCE): A randomised, double-blind, placebo-controlled, phase 3 trial. Lancet 2017;389:56-66.

11. Abou-Alfa GK, Meyer T, Cheng AL, et al Cabozantinib in patients with advanced and progressing hepatocellular carcinoma. N Engl J Med 2018;379:54-63.

12. Zhu AX, Kang YK, Yen CJ, et al. Ramucirumab after sorafenib in patients with advanced hepatocellular carcinoma and increased $\alpha$-fetoprotein concentrations (REACH-2): A randomised, double-blind, placebocontrolled, phase 3 trial. Lancet Oncol 2019;20:282-96.

13. El-Khoueiry AB, Sangro B, Yau T, et al. Nivolumab in patients with advanced hepatocellular carcinoma (CheckMate 040): An open-label, non-comparative, phase $1 / 2$ dose escalation and expansion trial. Lancet 2017;389:2492-502.

14. Zhu AX, Finn RS, Edeline J, et al. Pembrolizumab in patients with advanced hepatocellular carcinoma previously treated with sorafenib (KEYNOTE-224): A non-randomised, open-label phase 2 trial. Lancet Oncol 2018;19:940-52.

15. Finn RS, Ryoo BY, Merle P, et al. Pembrolizumab As Second-Line Therapy in Patients with Advanced Hepatocellular Carcinoma in KEYNOTE-240: A Randomized, Double-Blind, Phase III Trial. J Clin Oncol 2020;38:193-202.

16. Yau T, Hsu C, Kim TY, et al. Nivolumab in advanced hepatocellular carcinoma: Sorafenib-experienced Asian cohort analysis. J Hepatol 2019;71:543-52. 